\title{
Investigating the Energy Efficiency of TD-LTE Private Network
}

\author{
Ying Sun ${ }^{1,2, ~ a ~}$, Yuqing Zhong ${ }^{1, b}$, Xuanni Lin ${ }^{1, c}$ \\ ${ }^{1}$ Guangzhou Power Supply Co. Ltd., CSG, Guangzhou 510620, China \\ ${ }^{2}$ Shenzhen Graduate School of Harbin Institute of Technology, Shenzhen 518055, China \\ asycsggz@163.com, bzhongyq@guangzhou.csg.cn, 'linxuannishelly@qq.com
}

Keywords: wireless network; private network; TD-LTE; energy efficiency;

Abstract. TD-LTE private network is embracing unprecedented popularity due to its support of broadband services and ease deployment. However, due to the limited applications of power industry and the more urgent bandwidth requirements of telecommunication industries, the availabe bandwidth TD-LTE private network could be granted with is still insufficient and very limited. This paper investigates the spectral and energy efficiency performance of TD-LTE private network, thus providing references and suggestions for the system optimization and performance enhancement.

\section{Introduction}

Smart Grid is penetrating into all corners of people's daily life. Of the various communication methods such as optical fiber, power line or wireless network, private TD-LTE network is a long term evolution (LTE) based dedicated wireless broadband system and is flexible to implement and deploy, cost-effective and efficient compared with other communication networks, thus attracting lots of attentions from industry.

However, the broadband characteristic of TD-LTE private network is extremely limited due to the narrow bandwidth available for power industry. Meanwhile, with the trend of green communications, the energy consumption of the information and communication technology also requires careful design and control to reduce its impacts on the environment[1]. Therefore, the spectral and energy efficiency, namely, SE and EE, are two essential parameters that could reflect the broadbandness and greenness of a wireless sytem, which are the focus of this paper.

\section{Network Architecture}

There are two frequency bands available in China for TD-LTE private network, namely, $230 \mathrm{MHz}$ and $1.8 \mathrm{GHz}$, both of which share the same network architecture[2-3], as illustrated in Fig.1.

\section{Evaluation Parameter}

This section introduces parameters for evaluating the bandwidth utilization and power consumption.

Spectral Efficiency. Spectral efficiency, $\eta_{\mathrm{SE}}$, is the ratio of system capacity $C$ and bandwidth $B$, and is measured in bits/s/Hz. SE is used to evaluate the bandwidth utilization and is the transmission rate per unit bandwidth.

Energy Efficiency. Spectral efficiency, $\eta_{\mathrm{EE}}$, is the ratio of system capacity $C$ and bandwidth $E$, and is measured in bit/J. EE is used to evaluate the power consumption performance and is the transmitted bits per unit bandwidth.

\section{Performance Analysis}

This section introduces parameters for evaluating the bandwidth utilization and power consumption. 


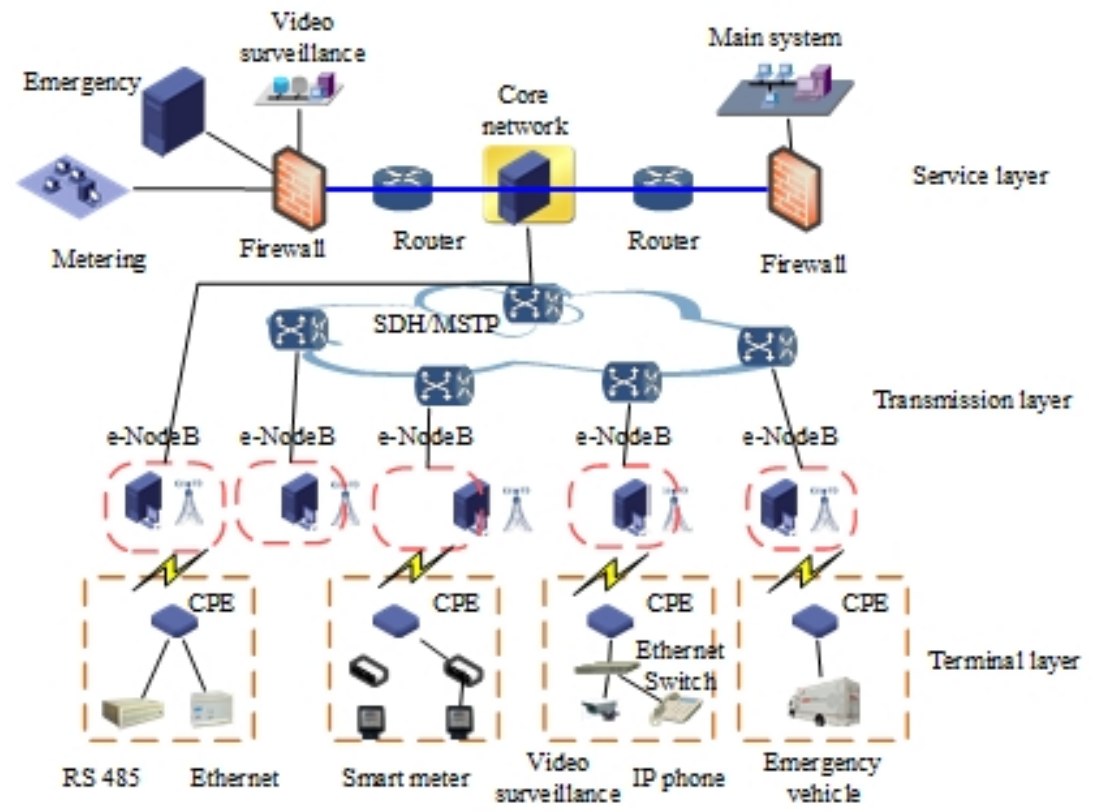

Figure 1. Network architecture of TD-LTE private network.

\section{Performance Analysis}

This section introduces parameters evaluating the bandwidth utilization and power consumption.

Single Cell Coverage. For single cell coverage, let $R$ be the transmission rate, $B$ the system bandwidth, $P_{\mathrm{T}}$ is the transmitting power, $\sigma^{2}$ is the average power of the additive white gaussian noise (AWGN), the upper bound to the capacity of the link could be calculated by

$$
R=B \log _{2}\left(1+\frac{P_{\mathrm{T}}}{B \sigma^{2}}\right) .
$$

SE could be obtained as

$$
\eta_{\mathrm{SE}}=\log _{2}\left(1+\frac{P_{\mathrm{T}}}{B \sigma^{2}}\right)
$$

EE could be obtained as

$$
\eta_{\mathrm{EE}}=\frac{\log _{2}\left(1+\frac{P_{\mathrm{T}}}{B \sigma^{2}}\right)}{P_{\mathrm{tot}}} .
$$

where $P_{\text {tot }}$ is the total consumed power.

When considering transmitting power as the only source of the total power consumption, i.e., $P_{\mathrm{tot}}=P_{\mathrm{T}}$, the relationship between $\eta_{\mathrm{SE}}$ and $\eta_{\mathrm{EE}}$ is

$$
\eta_{\mathrm{EE}}=\frac{\eta_{\mathrm{SE}}}{\left(2^{\eta_{\mathrm{SE}}}-1\right) B \sigma^{2}}
$$

When considering both transmission power and circuit power as the source of the total power consumption, i.e., $P_{\text {tot }}=P_{\mathrm{T}}+P_{\mathrm{C}}$, the relationship between $\eta_{\mathrm{SE}}$ and $\eta_{\mathrm{EE}}$ is

$$
\eta_{\mathrm{EE}}=\frac{\eta_{\mathrm{SE}}}{\left(2^{\eta_{\mathrm{SE}}}-1\right) B \sigma^{2}+P_{\mathrm{C}}}
$$


As can be seen, when only considering transmitting power, $\eta_{\mathrm{SE}}$ is inversely proportional to $\eta_{\mathrm{EE}}$, which means, when $\eta_{\mathrm{SE}}=0$, i.e., no data is transmitted, $\eta_{\mathrm{EE}}$ increases to infinity since there is no power consumption. On the other hand, when considering both transmitting and circuit power, the relationship between $\eta_{\mathrm{SE}}$ and $\eta_{\mathrm{EE}}$ appears as the bell curve illustrated in Fig.2.

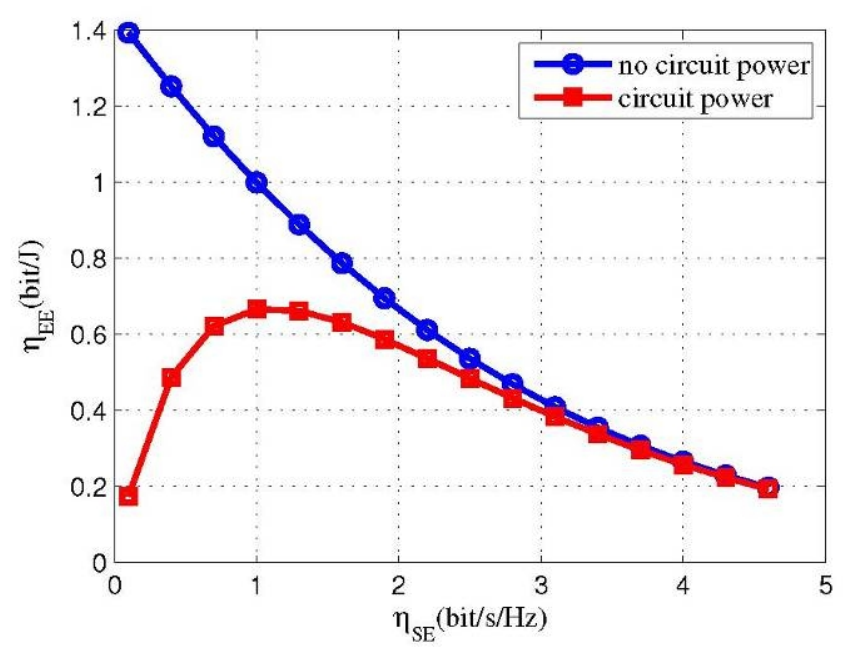

Figure 2. SE vs. EE in single cell coverage.

Multi-cell Coverage. For multi-cell coverage scenarios, inter-cell interference would affect the $\eta_{\mathrm{SE}}$ and $\eta_{\mathrm{EE}}$ performance. Assuming two cells, $c_{1}, c_{2}$, in a coverage area connect with two terminals, $u_{1}, u_{2}$, respectively. Meanwhile, two interference factors, i.e., $\alpha$ and $\beta$, are defined to denote the interference between $c_{1}, c_{2}$ and $u_{1}, u_{2}, \alpha$ and $\beta$ are calculated as follows:

$$
\begin{gathered}
\alpha=\frac{h_{c_{1}, u_{1}}}{h_{c_{2}, u_{1}}} . \\
\beta=\frac{h_{c_{2}, u_{2}}}{h_{c_{1}, u_{2}}} .
\end{gathered}
$$

where $h_{c_{1}, u_{1}}$ and $h_{c_{1}, u_{2}}$ are the channel gain between $c_{1}$ and $u_{1}, u_{2}$, respectively. $h_{c_{2}, u_{1}}$ and $h_{c_{2}, u_{2}}$ are the channel gain between $c_{2}$ and $u_{1}, u_{2}$, respectively.

Then, EE could be calculated as

$$
\eta_{\mathrm{EE}}=\frac{\eta_{c_{1}, \mathrm{SE}}+\eta_{c_{2}, \mathrm{SE}}}{P_{c_{1}, \mathrm{tot}}+P_{c_{2}, \mathrm{tot}}}=\frac{\eta_{c_{1}, \mathrm{SE}}+\eta_{c_{2}, \mathrm{SE}}}{2 P_{\mathrm{C}}+\Delta p P_{\mathrm{T}}\left(\frac{2^{\eta_{c_{1}, \mathrm{SE}}}-1}{\alpha}+\frac{2^{\eta_{c 2, \mathrm{SE}}}-1}{\beta}\right)}
$$

where $\eta_{c_{1}, \mathrm{SE}}$ and $\eta_{c_{2}, \mathrm{SE}}$ are the SE of $c_{1}$ and $c_{2}$, respectively; $\Delta p$ is the load-dependent power adjusting factor.

Based on the above analysis, the relationship between $\eta_{c_{1}, \mathrm{SE}}, \eta_{c_{2}, \mathrm{SE}}$ and $\eta_{\mathrm{EE}}$ is obtained.

No Inter-cell Interference. As illustrated in Fig.3, when there is no inter-cell interference (for instance, two cells are further apart or use different frequencies, $\alpha \rightarrow \infty, \beta \rightarrow \infty)$, SE enhancement of the two cells would improve the overall system EE.

Equal Inter-cell Interference. When there exists equal inter-cell interference (for instance, two cells are neighbouring and use the same frequency, $\alpha=5, \beta=5$ ), SE enhancement of either cell would cause interference to the other cell and thus affects the improvement of the overall system EE as like the bell plane illustrated in Fig.4. 


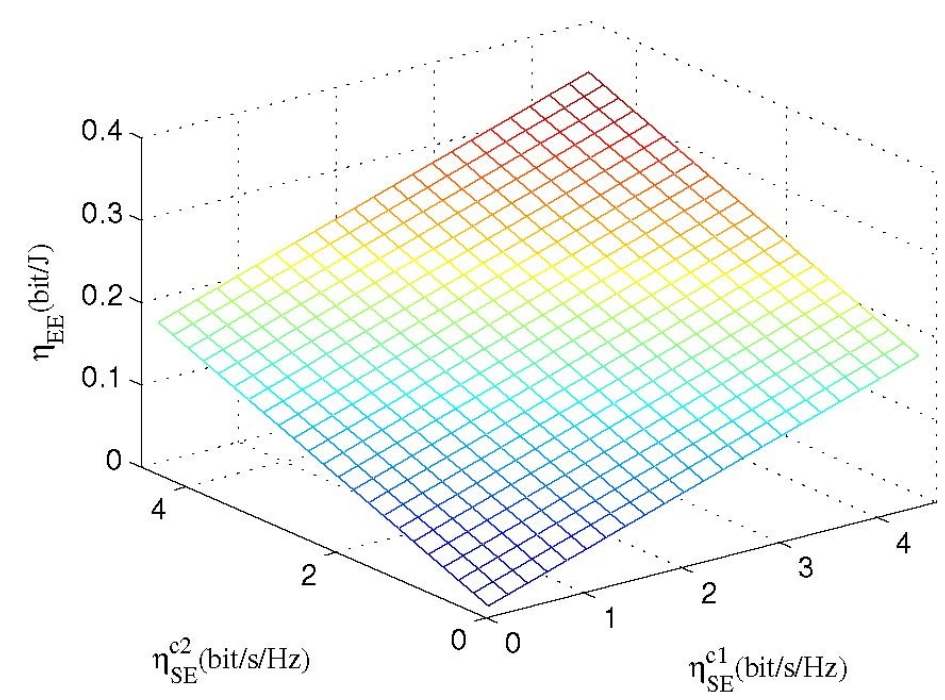

Figure 3. SE vs. EE in double cell coverage without inter-cell interference.

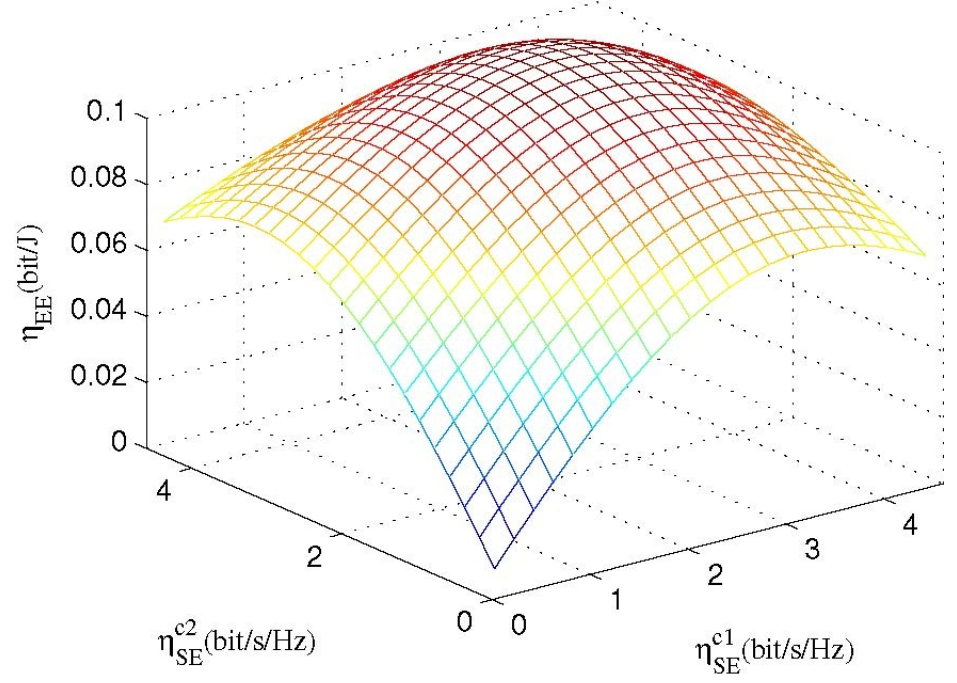

Figure 4. SE vs. EE in double cell coverage with equal inter-cell interference.

\section{Summary}

This paper investigated the bandwidth utilization and power consumption problem in TD-LTE private network. Two performance evaluation parameters, namely, SE and EE, were introduced to demonstrate the relationship between SE/EE performance and inter-cell interference, thus facilitating the network planning, deployment and optimization of TD-LTE private network in Smart Grid..

\section{References}

[1] A. Domenico, E. Strinati, A. Capone, Enabling green cellular networks: a survey and outlook, Computer Communications, 37(1): 5-24, 2014.

[2] Y. Sun, Y. Huang, L. Chen, A centralized LTE private wireless network architecture for smart grid communication network, Appl. Mech. Mater., 687(1):2363-2366, 2014.

[3] Y. Sun, X. Lin, L. Chen, Utilizing indicator computing assistant to facilitate distribution automation in LTE wireless private network, Appl. Mech. Mater., 691(1):3239-3242, 2014. 